\title{
Hipernatremia grave em recém-nascido resultante de falha no aleitamento materno: relato de caso
}

\section{Severe hypernatremia in newborn resulting from breastfeeding failure: case reports}

Bárbara Hellen de Sousa Cavalcante ${ }^{1}$, Thaís Moreira Lara², Arthur Machado Gonçalves ${ }^{3}$, Carlos Alberto Bhering ${ }^{4}$, Christianne Terra de Oliveira Azevedo ${ }^{5 *}$

Como citar esse artigo. Cavalcante,

B. H. S; Lara, T. M; Gonçalves, A. M; Bhering, C. A; Azevedo, C. T. O. Hipernatremia grave em recém-nascido resultante de falha no aleitamento materno: relato de caso. Revista de Saúde 2021 Ago/Nov.; 12 (3): 75-79.

\section{Resumo}

A hipernatremia é um distúrbio hidroeletrolítico definido por concentração sérica de sódio superior a $145 \mathrm{mEq} / \mathrm{L}$, sendo considerada grave quando superior a $160 \mathrm{mmol} / \mathrm{L}$. É uma condição rara em recém-nascidos, causando sintomas predominantemente neurológicos, que pode ocorrer pela alta ingestão de sódio, perda excessiva de líquidos ou diminuição da ingestão de líquidos. A hipernatremia grave tem sido especialmente associada à dificuldade na amamentação e à perda de peso. Existem alguns fatores de risco associados como primiparidade, parto cesáreo, peso materno excessivo, problemas de amamentação, falta de experiência em amamentação e baixa escolaridade materna. O objetivo deste trabalho foi apresentar um relato de caso de hipernatremia resultante de uma amamentação inadequada. O caso relatou um paciente da pediatria, de 23 dias de vida, apresentando quadro de desidratação grave e desnutrição, devido a dificuldades no aleitamento materno, sendo necessária a internação na Unidade de Terapia Intensiva (UTI). Após a realização de exames complementares, foi constatada hipernatremia $\left(\mathrm{Na}^{+} 182 \mathrm{mmol} / \mathrm{L}\right)$ sendo tratada com infusão de soro fisiológico $0,9 \%$. Paciente ainda evoluiu com convulsão, sendo tratada com fenobarbital. Após 36 dias de tratamento, neonato recebeu alta hospitalar. Portanto, conclui-se que a hipernatremia grave associada à dificuldade na amamentação pode ser uma condição evitada se o aleitamento materno for bem-sucedido, condição essa que seria possível com estratégias preventivas pelo sistema de saúde para gestantes e puérperas, como o acompanhamento mãe/neonato na primeira semana de vida.

Palavras-chave: Hipernatremia; Aleitamento Materno; Pediatria; Relato de Caso.

\begin{abstract}
Hypernatremia is a hydroelectrolytic disorder defined by a serum sodium concentration greater than $145 \mathrm{mEq} / \mathrm{L}$ being considered severe when greater than $160 \mathrm{mmol} / \mathrm{L}$. It is a rare condition in newborns, causing predominantly neurological symptoms, which can occur due to high sodium intake, excessive loss of fluids or decreased fluid intake. Severe hypernatremia has been especially associated with difficulty breastfeeding and weight loss. There are some risk factors associated with it such as primiparity, cesarean delivery, excessive maternal weight, breastfeeding problems, lack of experience in breastfeeding and low maternal education. The aim of this study was to present a case report of hypernatremia resulting from inadequate breastfeeding. The case reported a 23-day-old pediatric patient with severe dehydration and malnutrition due to difficulties in breastfeeding, requiring admission to the Intensive Care Unit (ICU). After complementary exams, hypernatremia $\left(\mathrm{Na}^{+} 182 \mathrm{mmol} / \mathrm{L}\right)$ was found and treated with $0.9 \%$ saline infusion. The patient also evolved with seizure and was treated with phenobarbital. After 36 days of treatment the neonate was discharged. Therefore, it is concluded that severe hypernatremia associated with difficulty in breastfeeding can be a prevented condition if breastfeeding is successful, something that would be possible with preventive measures by the health system for pregnant women and puerperal women, such as mother/newborn monitoring in the first week of life.

Keywords: Hypernatremia; Breastfeeding; Pediatrics; Case Reports
\end{abstract}

\section{Introdução}

A hipernatremia é um distúrbio hidroeletrolítico, onde há um déficit de água em relação ao sódio $\left(\mathrm{Na}^{+}\right)$ corporal total, definido laboratorialmente por uma concentração sérica de sódio superior a $145 \mathrm{mEq} / \mathrm{L}^{1}$. Pode ocorrer em recém-nascidos pela alta ingestão de sódio, perda excessiva de líquidos ou diminuição da ingestão de líquidos ${ }^{2,3}$, apresentando sinais predominantemente neurológicos, que podem ser inicialmente inespecíficos, como agitação, rigidez, letargia e sonolência. É possível ainda existirem outros sintomas correlacionados, sendo eles: estado de hidratação deficiente, diminuição da produção de urina, icterícia e convulsões epilépticas ${ }^{4}$, que ocorrem em até $40 \%$ das situações agudas de hipernatremia ${ }^{1}$, os quais podem evoluir para o coma e causarem complicações permanentes. ${ }^{4}$

A hipernatremia é considerada uma doença grave quando os níveis de $\mathrm{Na}^{+}$são superiores a $160 \mathrm{mmol} / \mathrm{L}$, podendo a mortalidade ser superior a $10 \%$ nesses $\operatorname{casos}^{1}$. 
Devido a maiores perdas insensíveis de água e sua incapacidade de comunicação quanto à necessidade de fluidos, as crianças são mais vulneráveis a esta condição do que os indivíduos mais velhos, sendo a perda excessiva de água por gastroenterite ou infecção sistêmica a causa mais comum em crianças mais velhas ${ }^{5}$. Entretanto, no recém-nascido a termo, a hipernatremia grave não só é considerada rara, como também é subestimada ${ }^{2}$.

Um estudo realizado na Irlanda, em neonatos com aleitamento materno exclusivo com até 29 dias de vida e mais de 34 semanas de gestação - que apresentavam durante a internação sódio sérico $\geq 160 \mathrm{mmol} / \mathrm{L}-$, demonstrou que a incidência de hipernatremia severa foi de 7 a cada 100.000 nascidos vivos (62 casos em 880.000 nascimentos). Já no estudo de Peñalvier Giner e cols, a incidência foi de 1,2 casos a cada 100 recémnascidos atendidos no período de $6 \operatorname{anos}^{6,7}$.

Dessa forma, a hipernatremia grave tem sido especialmente associada à dificuldade na amamentação e à perda de peso $^{4,8}$. Além disso, alguns fatores de risco são associados a ela, como: primiparidade, parto cesáreo, peso materno excessivo, problemas de amamentação, falta de experiência em amamentação e baixa escolaridade materna ${ }^{2,9,10}$. Então, é substancial definir inicialmente a causa da hipernatremia, visto que quando associada à amamentação, torna-se possível reduzir danos evitáveis e a aflição da hospitalização, além de poder aliviar o sofrimento materno e infantil ${ }^{6}$.

Outrossim, é importante lembrar que o leite materno é o único alimento essencial e exclusivo para o lactente até o $6^{\circ}$ mês de vida, sendo responsável pela promoção e proteção da saúde infantil1" ${ }^{11}$. Há sinais que podem ser observados para analisar se o bebê apresenta insuficiência no aleitamento, são eles: não ficar saciado após as mamadas, chorar muito, urinar menos vezes ao dia e evacuações infrequentes ${ }^{12}$. Sendo assim, é imprescindível acompanhar a mãe e o bebê nos primeiros dias de vida para que o aleitamento materno seja bem-sucedido e que complicações graves, como a hipernatremia, sejam evitadas ${ }^{13}$.

O objetivo deste trabalho foi apresentar um relato de caso de hipernatremia resultante de uma amamentação inadequada, e com isso ressaltar a importância do acompanhamento da mãe e neonato na $1^{\mathrm{a}}$ semana de vida como prioridade para o sistema de saúde, com o intuito de detecção precoce de condições evitáveis e corrigíveis, cujas consequências são muito graves.

\section{Relato de Caso}

K.V.S.C, feminino, 23 dias de vida, nascida de parto vaginal, idade gestacional de 39 semanas, APGAR 9/10, sem intercorrências. Mãe primípara procurou atendimento ambulatorial para a filha, relatando queixa de dificuldade para amamentar e desnutrição, sendo detectado quadro de desidratação grave, resultando no encaminhamento para internação hospitalar. Ao ser avaliado no hospital de referência, encontrava-se com quadro de desidratação grave e desnutrição, pesando $1910 \mathrm{~g}$, foram solicitados exames, iniciado antibiótico empiricamente (ampicilina + gentamicina) e dieta oral. Constatada a gravidade clínica do recém-nascido (RN), o mesmo foi encaminhado para internação em Unidade de Terapia Intensiva (UTI) neonatal.

$\mathrm{Na}$ admissão pela UTI, paciente apresentavase hipoativo, perfusão lentificada, fontanela anterior deprimida, rigidez e hipertonia de membros, sendo suspeitado sepse neonatal, além de desidratação. Sinais vitais: PA 77x56 mmHg, FC 127 bpm, SatO 95\%. Ao exame: paciente eupneico, afebril, com diurese concentrada e evacuações presentes, desnutrido, emagrecido, hipohidratado $(2+/ 4+)$, acianótico, pele seca, choroso ao manuseio, com ausência de saliva ou lágrimas. Ritmo cardíaco regular, em 2 tempos, bulhas normofonéticas e sem sopros. Murmúrio vesicular universalmente audível, sem ruídos adventícios. Abdome escavado, sem massas ou visceromegalias. Pulsos presentes, com amplitude preservada. Foi colhido líquor, sem intercorrências, e o exame de sangue constatou $\mathrm{Na}^{+}$ de $182 \mathrm{mmol} / \mathrm{L}$ (Tabela 1). Mantido antibioticoterapia e instalado hidratação com correção de água livre.

Tabela 1. Evolução dos valores do sódio sérico durante internação.

\begin{tabular}{c|c} 
Dia de internação & $\mathbf{N a}^{+}(\mathbf{m m o l} / \mathbf{L})$ \\
\hline $1^{\mathrm{o}}$ & 180 \\
\hline $2^{\mathrm{o}}$ às $9 \mathrm{~h}$ & 182 \\
\hline $2^{\mathrm{o}}$ às $13: 30 \mathrm{~h}$ & 179 \\
\hline $2^{\circ}$ às $19 \mathrm{~h}$ & 175 \\
\hline $3^{\mathrm{o}}$ às $8 \mathrm{~h}$ & 162 \\
\hline $3^{\circ}$ às $19 \mathrm{~h}$ & 154 \\
\hline $4^{\mathrm{o}}$ & 150 \\
\hline $8^{\mathrm{o}}$ & 151 \\
\hline $5^{\mathrm{o}}$ & 136 \\
\hline
\end{tabular}


No dia seguinte, paciente evoluiu mantendo hipoatividade, pouco reativo, acianótico, anictérico, hidratado - com saliva fluída -, desnutrido, pálido e não realizando a abertura dos olhos. Apresentou sucção negativa, além de reflexos de preensão palmar, plantar e moro ausentes. Exame apontou hipernatremia de 179 $\mathrm{mmol} / \mathrm{L}$, apesar da reposição que nesse momento foi ajustada, plaquetas de $9.000 \mathrm{mil} / \mathrm{mm}^{3}$, indicando uma plaquetopenia e distúrbio de coagulação, tratada com concentrado de plaquetas e plasma fresco. Exames de imagem foram feitos, com os seguintes resultados: a ultrassonografia (USG) transfontanela apresentou hemorragia intracraniana grau 1 e a USG abdominal indicou paredes de vesícula espessada, além de líquido livre em cavidade. Neste mesmo dia, foi colhido sangue para oTORCHS (toxoplasmose, outras-HIV,HBV, sífilis -, rubéola, citomegalovírus e herpes simples) materno, o qual apresentou-se negativo. Foram também coletados exames do RN para afastar infecções congênitas como citomegalovírus (CMV), toxoplasmose, rubéola, sífilis, herpes simples e erros inatos do metabolismo, além da hemocultura que também se apresentou negativa.

No terceiro dia de internação, foi colhido novo exame de sangue (Tabela 1), apresentando $\mathrm{Na}^{+}$de $162 \mathrm{mmol} / \mathrm{L}$, com manutenção da infusão de soro fisiológico $0,9 \%$. Neste momento, o paciente encontrava-se hemodinamicamente estável, aceitando dieta normalmente, apresentava pele descamativa, que poupava face, palmas de mãos e plantas dos pés. No quarto dia de internação, apresentou convulsão tônicoclônica generalizada, sendo tratada com fenobarbital de ataque.

No decorrer dos dias, ao realizar um hemograma completo foi constatado leucocitose de $16500 / \mathrm{mm}^{3}$, tornando o diagnóstico de sepse provável. Houve também uma nova coleta do teste do pezinho para fibrose cística, o qual descartou a doença. Posteriormente, foram realizados os testes TORCH da mãe, constatando CMV IgG positivo e IgM negativo, toxoplasmose IgM e IgG negativos além de rubéola IgG positivo e IgM negativo. Ademais, foi solicitado parecer da neuropediatra, que orientou quanto a manutenção do fenobarbital, apenas com a redução da dose para $3 \mathrm{mg} / \mathrm{kg} / \mathrm{dia}$. No oitavo dia de internação, o paciente evoluiu com normalização do sódio (Tabela 1).

O paciente continuou sendo monitorado nos dias seguintes, tendo ocorrido a realização de eletroencefalograma (EEG) - que mostrou ritmo lentificado e ondas agudas em região centro parietal esquerda com generalização secundária -, sendo solicitado novo parecer da neuropediatria, que orientou aumentar o fenobarbital para $4 \mathrm{mg} / \mathrm{kg} / \mathrm{dia}$.

Outrossim, suspeitou-se de CMV, sendo descartado após a realização de PCR em urina com resultado negativo. Em relação à herpes, o resultado obtido foi IgG positivo e IgM negativo.
Neonato continuou sob os cuidados da equipe, com diagnóstico de hipernatremia grave, desnutrição e provável sepse, evoluindo com dieta oral plena através do aleitamento materno durante os últimos 10 dias de internação, permanecendo sem intercorrências e obtendo alta após 36 dias de internação com peso de $3.675 \mathrm{~kg}$.

\section{Discussão}

A hipernatremia é uma condição grave no período neonatal ${ }^{8}$, que quando relacionada à amamentação era considerada incomum, porém a incidência vem aumentando e a falha em corrigi-la pode resultar em uma alta taxa de mortalidade ${ }^{15,16}$. Ocorre, principalmente, em mães primíparas com dificuldade em amamentar o bebê, podendo causar produção e secreção inadequada de leite materno ${ }^{17}$. A perda ponderal maior do que $7 \%$ durante a primeira semana de vida deve constituir um sinal de alerta quanto à possibilidade de que o aleitamento materno seja insuficiente, devendo ser avaliado o estado clínico do neonato ${ }^{18}$.

Crianças com hipernatremia geralmente aparecem agitadas e irritadas, podendo progredir para letargia, apatia e coma. O exame neurológico pode revelar rigidez de nuca, aumento do tônus, mioclonia, podendo ainda apresentar crises convulsivas tônico-clônicas e de ausência ${ }^{19}$, sendo a gravidade destes sintomas relacionada não só ao grau de hiperosmolaridade, mas principalmente à velocidade com que esta se instalou. Os sintomas são devidos às alterações no conteúdo da água cerebral ${ }^{20}$.

No caso relatado, o neonato encontrava-se ao exame hipertônico, rígido, desnutrido e com perda de quase $50 \%$ do peso corporal do nascimento. Diagnosticado inicialmente com quadro grave de desidratação, desnutrição e após resultado do exame sanguíneo colhido apresentou $\mathrm{Na}^{+}$corporal de 182 $\mathrm{mmol} / \mathrm{L}$, o qual constatou a hipernatremia grave, que começou a ser tratada com reposição de água livre $10 \mathrm{ml} /$ $\mathrm{kg}$, havendo queda inicial de $10 \mathrm{mEq} / \mathrm{L}$ em 4 horas.

O objetivo da reposição de água livre é diminuir o sódio no sangue em cerca de $12 \mathrm{mEq} / \mathrm{L}$ a cada 24 horas, chegando a uma taxa de $0,5 \mathrm{mEq} / \mathrm{L} / \mathrm{h}$. A hipernatremia em si pode causar convulsões, como ela também pode ocorrer durante o tratamento, especialmente durante a ressuscitação com fluidos. É importante que ocorra a monitorização frequente do sódio sérico para que a terapia hidroeletrolítica seja ajustada, proporcionando uma correção, nem muito lenta e nem muito rápida ${ }^{21}$.

A crise tônico-clônica generalizada é definida como contrações musculares mantidas (tônicas) em todo o corpo, seguida de contrações alternadas por um breve relaxamento, rítmicas e repetitivas (clônicas) ${ }^{22}$, são raras no $\mathrm{RN}$, sendo a hipernatremia uma das principais 
etiologias $^{23}$. O paciente do caso relatado, no dia 4 de internação, apresentou tal convulsão, sendo iniciado o fenobarbital, que é a primeira droga de escolha para essa faixa etária ${ }^{24}$. O EEG tem papel fundamental na investigação neurológica e imprescindível para diagnóstico de síndromes epilépticas ${ }^{25}$. Este foi realizado e apresentou ritmo lentificado e ondas agudas em região centro parietal esquerda com generalização secundária. As crises que se iniciam no lobo parietal se caracterizam por alterações sensitivas e costumam evoluir para crises tipo grande $\mathrm{mal}^{26}$. Neonato apresentou crise única sem mais alterações e complicações.

A USG Transfontanela apresentou hemorragia intracraniana grau 1 , que se refere à hemorragia apenas ao nível da matriz germinativa subependimária ${ }^{27}$, a qual precisa ser monitorada ao longo dos dias, geralmente sem grandes complicações ${ }^{28}$.

A hemocultura e o líquor devem ser colhidos na suspeita de sepse, sendo a hemocultura considerada o teste ideal e padrão ouro para diagnóstico de infecção de corrente sanguínea e bacteremia ${ }^{24}$. De acordo com a Agência Nacional de Vigilância Sanitária (ANVISA), a sepse é definida como uma resposta sistêmica, sem outra causa reconhecida que não a infecciosa, associada a dois ou mais dos seguintes sinais e sintomas: bradicardia, instabilidade térmica, intolerância alimentar, hipoatividade, apneia, piora do desconforto respiratório, intolerância a glicose, letargia e instabilidade hemodinâmica ${ }^{23}$. No caso relatado, a hemocultura foi negativa em todos os testes e o líquor também, os quais não confirmaram a sepse.

Portanto, é claro que em casos de hipernatremia a reidratação deve ser realizada imediatamente, podendo ser oral com leite materno expresso ou amamentação direta ou $100 \mathrm{ml} / \mathrm{kg} /$ dia de reidratação hídrica, devendo ser lenta, pois a correção rápida do aumento da concentração sérica de $\mathrm{Na}^{+}$predispõe a mudanças osmóticas no cérebro que podem exacerbar problemas neuronais.

\section{Conclusão}

A hipernatremia grave associada ao aleitamento materno é uma condição subdiagnosticada, capaz de causar danos irreversíveis ao neonato, quando não reconhecida precocemente. Está relacionada, principalmente, à falta de conhecimento de mães inexperientes sobre amamentação e ao insuficiente suporte dos profissionais de saúde. A melhoria na educação de gestantes e puérperas sobre amamentação e conselhos sobre sinais de alerta de desidratação e inadequação da amamentação seriam intervenções capazes de evitar esta condição.

\section{Comitê de Ética}

O relato apresentado foi aprovado pelo Comitê de Ética e Pesquisa da Universidade de Vassouras, parecer $\mathrm{n}^{\mathrm{o}}$ 4.643.776, CAAE 44299121.1.0000.5290, de 12 de abril de 2021.

\section{Referências}

1. Sousa V, Carrusca C, Santos M. Desidratação hipernatrémica no recém-nascido. Nascer e Cresce 2016;25(1):22-26.

2. Lavagno C, Camozzi P, Renzi S, Lava SA, Simonetti GD, Bianchetti MG, Milani GP. Breastfeeding-Associated Hypernatremia: A Systematic Review of the Literature. J Hum Lact 2016;32(1):67-74

3. Laing IA, Wong CM. Hypernatraemia in the first few days: is the incidence rising? Arch Dis Child Fetal Neonatal Ed 2002;87:158-162.

4. Oddie S, Richmond S, Coulthard M. Hypernatraemic dehydration and breast feeding: a population study. Arch Dis Child 2001;85(4):318-320.

5. Somers MJ, Hypernatremia in children Authors: Michael J Somers, Traum AZ. Hypernatremia in children. Up To Date; 2020 [acesso em $1 \mathrm{dez}$ 2020]. Disponível em: https://www.uptodate.com/contents/hypernatremiain-children

6. Oddie SJ, Craven V, Deakin K, Westman J, Scally A. Severe neonatal hypernatraemia: a population-based study. Arch Dis Child Fetal Neonatal Ed 2013;98(5):F384-7

7. López-Candiani C, Salamanca-Galicia O. Hipernatremia en 79 recién nacidos. Factores asociados a desenlace adverso. Acta Ped Mex 2012;33(5):239-245.

8. Mujawar NS, Jaiswal AN. Hipernatremia no recém-nascido: hipernatremia neonatal e desidratação hipernatrêmica em recém-nascidos que recebem aleitamento materno exclusivo. Indian J Crit Care Med 2017;21(1):30-33

9. Ferrández-González M, Bosch-Giménez V, López-Lozano J, MorenoLópez N, Palazón-Bru A, Cortés-Castell E. Weight loss thresholds to detect early hypernatremia in newborns. J Ped 2019;95(6):689-695.

10. Vieira GO, Silva LR, Vieira TO, Almeida JAG, Cabral VA. Hábitos alimentares de crianças menores de 1 ano amamentadas e não-amamentadas. J Ped 2004;80(5):411-416

11. Manual Brasil; Ministério da Saúde; Secretaria de Atenção Primaria à Saúde; Departamento de Promoção da Saúde. Guia alimentar para crianças brasileiras menores de 2 anos. Brasília: Ministério da Saúde, 2019.

12. Ministério da Saúde; Secretaria de Atenção à Saúde; Departamento de Atenção Básica. Saúde da criança: aleitamento materno e alimentação complementar. Brasília, DF: O Ministério; 2015.

13. Bischoff AR, Dornelles AD, Carvalho CG Treatment of Hypernatremia in Breastfeeding Neonates: A Systematic Review. Biomed Hub 2017;27;2(1):1-10.

14. Domínguez RRB; Roncero AI; Cid JLH; Ramírez CS. Hipernatremia grave: supervivencia sin secuelas neurológicas. Anales de Pediatría 2003;58(4):376-380.

15. Suliman OS. Dying for milk: A neonate with severe hypernatremia associated with inadequate breast feeding. Sudan J Paediatr 2015;15(2):5562.

16. Çaglar MK, Özer I, Altugan FS. Fatores de risco para perda de excesso de peso e hipernatremia em bebês amamentados exclusivamente. Braz J Med Biol Res 2006; 39(4):539-544.

17. Gomes SM, Fernandes CA, Ramos H, Fernandes E, Santos M, Nascimento O, et al. Desidratação hipernatrêmica associada ao aleitamento materno. Einstein 2009:7(2):206-10. 
18. Moritz ML, Ayus JC. Disorders of water metabolism in children: hyponatremia and hypernatremia. Pediatr Rev 2002;23(11):371-80.

19. Abreu CP. Hipernatremia: uma revisão. Medicina Interna 2002;9(2):100-110.

20. Nelson WE, Kliegman RM, Jenson HB, Behrman RE. Tratado de Pediatria. $18^{\text {a }}$ ed. Rio de Janeiro: Elsevier; 2009.

21. Brito AR, Vasconcelos MM, Almeida SSA. Convulsões. Revista de Pediatria SOPERJ 2017;17(supl 1)(1):56-62.

22. Meireles LA, Vieira AA, Costa CR. Avaliação do diagnóstico da sepse neonatal: uso de parâmetros laboratoriais e clínicos como fatores diagnósticos. Rev Esc Enferm USP 2011; 45(1):33-39.

23. Procianoy RS, Silveira RC. Os desafios no manejo da sepse neonatal. J Pediatr 2020;96(suppl 1):80-86.

24. Silva DF, Lima MM. Aspectos gerais e práticos do EEG. Rev Neurociênc 1998;6(3):137-146.

25. Rizzutti S, Muszkat M, Campos CJR. Monitorização eletroencefalográfica ambulatorial na epilepsia de difícil controle da infância. Arq Neuro-Psiquiatr 2001;59(4):875-883.

26. Silva LR, Iser BPM, Tartare B, Bonetti HS. Aspectos perinatais relacionados à hemorragia intracraniana em recém-nascidos de muito baixo peso no Sul do Brasil. Rev Bras Ginecol Obstet 2015;37(4):159-163.

27. Nevs LAT, Chehuen N JA, Kneipp D, Fonseca LG, Rosado MP, Barreto MRP. Hemorragia Intracraniana no recém-nascido pré-termo. Casuística da UTI Neonatal do Hospital Albert Sabin. HU Rev Juiz de Fora 2007;33(2):49-54

28. Mujawar NS, Jaiswal AN. Hipernatremia no recém-nascido: hipernatremia neonatal e desidratação hipernatrêmica em recém-nascidos que recebem aleitamento materno exclusivo. Indian J Crit Care Med 2017;21(1): 30-33. 